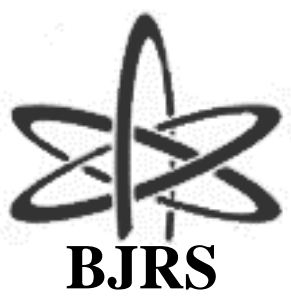

BRAZILIAN JOURNAL

$\mathrm{OF}$

RADIATION SCIENCES

01 (2013) 1-15

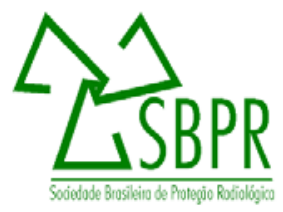

\title{
INTERNAL DOSIMETRY IN NUCLEAR MEDICINE
}

\author{
Michael G. Stabin ${ }^{\mathrm{a}}$ \\ ${ }^{a}$ Vanderbilt University, Nashville, TN USA
}

\begin{abstract}
This article reviews the current state of the art in the calculation of radiation doses for radiopharmaceuticals. Basics of dose calculations are briefly reviewed, and the sources of input data for the calculations are reviewed. Although dose calculations are needed for all kinds of radiopharmaceuticals, dose calculations in therapeutic applications are the most important for the patient; the status of dose calculations for most important therapeutic uses of radiopharmaceuticals is discussed, and the importance of performing patient-individualized dose calculations is emphasized
\end{abstract}

Keywords:

Internal Dosimetry, Dose Calculations, Nuclear Medicine

\section{INTRODUCTION}

Whenever patients are exposed to ionizing radiation, an estimation of radiation doses is an essential element of balancing the risks and benefits of the proposed examination. External sources of ionizing radiation in the healing arts include plain film x-rays, computed tomography (CT) examinations, positron emission tomography (PET), single photon emission tomography (SPECT), PET/CT, SPECT/CT and nuclear medicine studies. The calculation of radiation dose 
estimates from internal sources of radioactivity have evolved over many decades, from the simple thyroid self-dose estimates of Marinelli and Quimby [1, 2] to three dimensional dose maps and dose-volume histograms for individual organs and tumors [3]. Methods and tools exist for the calculation of radiation dose to important tissues of the body, for dose and risk assessment, but sadly, a decades-old mentality pervades the nuclear medicine community that calculation of radiation doses and optimization of therapy for individual patients in unnecessary. Doses are calculated to permit the approval of new radiopharmaceuticals, but once approved, the compounds are administered to patients using a fixed activity ( $\mathrm{MBq}$ ) or activity per unit body weight $(\mathrm{MBq} / \mathrm{kg})$ approach, with few exceptions. For diagnostic radiopharmaceuticals, this is reasonable and safe; misadministrations occur, but doses to any individual organ are not likely to exceed $50 \mathrm{mSv}$, and effective doses are low, compared to many radiation exposures in medical imaging. In therapeutic applications, however, our goal is to maximize the radiation dose to malignant tissues while sparing as much as possible the dose to normal, healthy tissues. This can only be accomplished by characterizing the uptake and clearance of activity in the various tissues of the body, which requires serial imaging of patients and quantitative analysis of the results, an effort which nuclear medicine physicians are mostly loathe to perform. Thus, patients are generally receiving suboptimal therapy, compared to patients receiving external beam therapy for cancer, for whom, careful radiation therapy plans are made on a patient-specific basis every day. An exception is the monoclonal antibody Bexxar, for which at least 'whole body' doses (as a surrogate for red marrow doses) are calculated to estimate a patient-individualized activity administration; many in the European community are also demonstrating how patientindividualized dose calculations are necessary for managing renal toxicity for patients treated for neuroendocrine tumors, as will be discussed below. Regulatory agencies, however, are basically ignoring calls for equal use of dosimetry in the treatment of cancer patients with either external or internal sources of ionizing radiation; this issue needs to be addressed. 


\section{COMPONENTS OF THE CALCULATION}

A 'generic' equation for the absorbed dose rate in an organ or tumor uniformly contaminated with radioactivity can be shown as:

$$
\dot{D}_{T}=\frac{k \sum_{S} A_{S} \sum_{i} n_{i} E_{i} \phi_{i}(T \leftarrow S)}{m_{T}}
$$

Where: $\quad \dot{D}=$ absorbed dose rate in target region $\mathrm{T}$ (rad/hr or Gy/sec)

$\mathrm{A}_{\mathrm{S}}=$ activity $(\mu \mathrm{Ci}$ or $\mathrm{MBq})$ in source region $\mathrm{S}$

$\mathrm{n}_{\mathrm{i}}=$ number of radiations with energy $\mathrm{E}_{\mathrm{i}}$ emitted per nuclear transition

$E_{i}=$ energy for the ith radiation emitted by the nuclide $(\mathrm{MeV})$

$\phi_{\mathrm{i}}(\mathrm{T} \leftarrow \mathrm{S})=$ fraction of energy emitted that is absorbed in the target region $\mathrm{T}$ originating in source region $\mathrm{S}$

$\mathrm{m}_{\mathrm{T}}=$ mass of target region $\mathrm{T}$ ( $\mathrm{g}$ or $\left.\mathrm{kg}\right)$

$\mathrm{k}=$ proportionality constant (rad-g/ $\mu \mathrm{Ci}-\mathrm{hr}-\mathrm{MeV}$ or $\mathrm{Gy}-\mathrm{kg} / \mathrm{MBq}-\mathrm{sec}-\mathrm{MeV}$ )

The proportionality constant must be properly calculated and applied for the unit system of choice. One may also apply radiation weighting factors (once called "quality factors") to the result of this equation to calculate the equivalent dose rate. For many years, this was not an important issue, when nuclear medicine involved only beta and gamma emitters; now the introduction of some alpha emitters makes this an important consideration. The value recommended by the International Commission on Radiological Protection (ICRP) for the radiation weighting factor for alpha emitters is 20 , but this is believed to be too high for use in calculating doses from nuclear medicine therapy agents [4]. 
We are usually interested in an estimate of total absorbed dose from an administration, rather than an initial dose rate. In equation 1 the quantity activity (nuclear transitions per unit time) causes the outcome of the equation to have time dependence. To calculate cumulative dose, the time integral of the dose equation must be calculated. In most cases, the only term which has time dependence is activity, so the integral is just the product of all of the factors in the above equation except for activity by the integral of the time-activity curve.

Figure 1: Activity x time

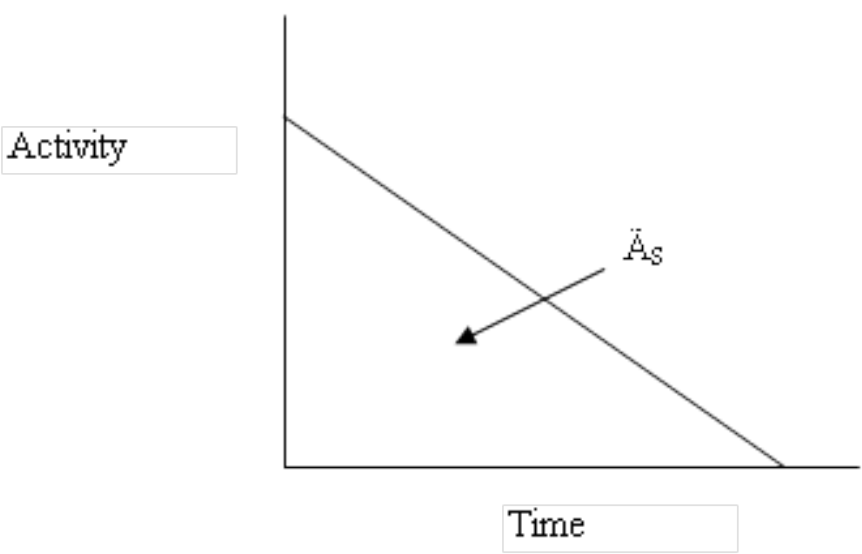

Regardless of the shape of the time-activity curve, its integral, however obtained, will have units of the number of total nuclear transitions (activity, which is transitions per unit time, multiplied by time). Therefore, the equation for cumulative dose would be shown as:

$$
D_{T}=\frac{k \sum_{S} \tilde{A}_{S} \sum_{i} n_{i} E_{i} \phi_{i}(T \leftarrow S)}{m_{T}}
$$

Where: $\quad \mathrm{D}_{\mathrm{T}}$ is the absorbed dose in target region $\mathrm{T}$ (rad or Gy) and $\tilde{\mathrm{A}}_{\mathrm{S}}$ is the cumulated activity in source region $\mathrm{S}$ ( $\mu \mathrm{Ci}$-hr or MBq-sec). 


\subsection{Input Data}

Some consider equation 2 to consist of two general parts, (1) a 'biological' component, involving $\tilde{A}$, and (2) a 'physical' part, involving all of the other terms. Indeed part (2) is often characterized by 'Dose Factors' for specified source and target regions for a particular radionuclide [5]. These Dose Factors are typically tabulated for retrieval, and/or integrated in software programs to facilitate dose calculations, e.g. OLINDA/EXM [6]. The values for the 'absorbed fractions' come from modeling assumptions or Monte Carlo calculations in anthropomorphic 'phantoms' (mathematical models of the human body). These phantoms for many years were based on geometrical constructs to represent the body and its organs [7, 8], but more recently have been replaced with more realistic models based on medical image data [9]. Thus calculation of radiation dose for a given radiopharmaceutical usually involves acquiring data to calculate the Âs values, which involve the biological distribution and retention of activity in the body . Data are obtained in animals or in human volunteers or patients. In the former case, the animals are given the radiopharmaceutical and may be sacrificed, and individual organs harvested and assayed for their radioactivity content, or they may be imaged using small animal PET or SPECT imaging systems. Human data may be gathered in anterior/posterior projection mode in SPECT systems, or using PET or SPECT imaging systems. Animal data need to be extrapolated for use in calculating dose estimates for humans [10]. For either human or animal data, gathering the correct number of data points at appropriate time intervals is essential to a successful analysis. Most kinetic data are characterized by one or more exponential terms. To characterize any one phase of uptake or clearance with an exponential function, one needs a minimum of two data points [11]; for example, if an organ has an early, fast clearance phase and a later, slow clearance phase, a minimum of four data points are needed, with two during the early phase and two during the late phase. Careful design of a study to collect biokinetic data, with these considerations, is essential to obtain the necessary data. 


\subsection{Diagnostic Radiopharmaceuticals}

There are many diverse radiopharmaceuticals employed in diagnostic imaging of nuclear medicine patients. The number and type of agents is constantly changing, so it is a challenge to maintain organized lists of the radiation dose estimates for all agents that may be in use. Dose estimates for diagnostic radiopharmaceuticals are not generally needed on a daily basis. When a new radiopharmaceutical is being considered for approval, part of the approval process involves characterization of the dosimetry for the agent, ultimately being based on data gathered in humans. These dose estimates are included in the standard information distributed with the agent (often referred to as the 'package insert'). Consideration of radiation dose is not an important issue in routine administrations for diagnostic imaging; the doses are generally low, and the main concern is to give the best amount of activity that will produce a good quality image. Radiation dose becomes a concern with a radiopharmaceutical misadministration occurs, when an administration occurs accidentally or intentionally to a pregnant woman, and in a few other limited circumstances. Dose estimate tables in 'package inserts' are not always the most reliable or up to date; compendia published by an ICRP Task Group on Radiopharmaceutical Dosimetry are generally considered to be the most reliable and up to date. They are currently contained in three publications that have been produced since 1983 [12].

For diagnostic, but not therapeutic, applications of radiopharmaceuticals, The ICRP also defines a quantity effective dose (E); certain organs or organ systems were assigned dimensionless 'tissue weighting factors', which are assumed to relate to their differing radiosensitivity for expressing fatal cancers or genetic defects. Summing the products of each organs actual equivalent dose and the assigned tissue weighting factors gives a single number, which is the 'effective dose' The most recent weighting factors (Table 1) were given in ICRP Publication 103 [13]. Effective dose values for a number of diagnostic radiopharmaceuticals given in these ICRP publications are shown in Table 2. 
Table 1. Weighting Factors Recommended by the ICRP for Calculation of Effective Dose [13]

\begin{tabular}{lc}
\hline Organ & Tissue Weighting Factor \\
\hline Gonads & 0.08 \\
Red Marrow & 0.12 \\
Colon & 0.12 \\
Lungs & 0.12 \\
Stomach & 0.12 \\
Bladder & 0.04 \\
Breasts & 0.12 \\
Liver & 0.04 \\
Esophagus & 0.04 \\
Thyroid & 0.04 \\
Skin & 0.01 \\
Bone Surfaces & 0.01 \\
Brain & 0.01 \\
Salivary glands & 0.01 \\
Remainder & 0.12 \\
\hline
\end{tabular}

\subsection{Therapeutic Radiopharmaceuticals}

Standardized dose estimates may be given for therapeutic radiopharmaceuticals (note the values in Table 2 for ${ }^{131}$ I-NaI, which may be given in tracer or therapeutic amounts, and for ${ }^{153} \mathrm{Sm}$-EDTMP), for general interest. When therapeutic radiopharmaceuticals are used, however, it is more appropriate to gather biokinetic data for each individual subject, using a diagnostic level of the therapeutic agent or surrogate (e.g. use of ${ }^{111} \mathrm{In}$-Zevalin to determine the probable biodistribution of ${ }^{90} \mathrm{Y}$-Zevalin), and performing a predictive dosimetry study to determine the maximum amount of the therapeutic agent that can be given while not exceeding undesirable radiation doses to healthy tissues. Such optimization of therapy is performed for every patient receiving external beam therapy for cancer, but unfortunately, is not often done to plan nuclear medicine therapy. Typically, standard amounts of activity or activity per unit body weight of the subjects is given, with no pre- or post-therapy evaluation of the radiation doses received. 
Table 2. Standardized Dose Estimates for Selected Radiopharmaceuticals [12].

\begin{tabular}{|c|c|}
\hline Radiopharmaceutical & Effective Dose (mSv/MBq) \\
\hline O-15 Water & 9.30E-04 \\
\hline F-18 Fluoro-deoxyglucose & 1.90E-02 \\
\hline Ga-67 Citrate & $1.00 \mathrm{E}-01$ \\
\hline I-123 Hippuran & $1.20 \mathrm{E}-02$ \\
\hline I-123 Sodium Iodide $\quad$ (25\% Uptake) & $1.70 \mathrm{E}-01$ \\
\hline I-125 Albumin & 2.20E-01 \\
\hline I-131 Hippuran & 5.20E-02 \\
\hline I-131 MIBG & $1.40 \mathrm{E}-01$ \\
\hline I-131 Sodium Iodide $\quad$ (25\% Uptake) & $1.70 \mathrm{E}+01$ \\
\hline In-111 DTPA - normal renal function & 2.10E-02 \\
\hline In-111 Pentetreotide also known as Octreoscan & $5.40 \mathrm{E}-02$ \\
\hline Sm-153 Lexidronam also know as Quadramet & $1.97 \mathrm{E}-01$ \\
\hline Tc-99m Disofenin also know as HIDA (iminodiacetic acid) & 1.70E-02 \\
\hline Tc-99m Exametazime also known as Ceretec and HMPAO & 9.30E-03 \\
\hline Tc-99m Macro aggregated albumin (MAA) & $1.10 \mathrm{E}-02$ \\
\hline $\begin{array}{l}\text { Tc-99m Medronate also know as Tc-99m Methyenedi- } \\
\text { phosphonate (MDP) }\end{array}$ & 5.70E-03 \\
\hline Tc-99m Mertiatide also know as MAG3 & 7.00E-03 \\
\hline Tc-99m Neurolite also known as ECD and Bicisate & 1.10E-02 \\
\hline Tc-99m Pentetate also know as Tc-99m DTPA & 4.90E-03 \\
\hline Tc-99m Pyrophosphate & 5.70E-03 \\
\hline Tc-99m Red Blood Cells & 7.00E-03 \\
\hline Tc-99 Sestamibi also know as Cardiolite (rest) & 9.00E-03 \\
\hline Tc-99 Sestamibi also know as Cardiolite (stress) & 7.90E-03 \\
\hline Tc-99m Sodium Pertechnetate & 1.30E-02 \\
\hline Tc-99m Sulfur Colloid & $9.40 \mathrm{E}-03$ \\
\hline Tc-99m Tetrofosmin also know as Myoview (rest) & 7.60E-03 \\
\hline Tc-99m Tetrofosmin also know as Myoview (stress) & 7.00E-03 \\
\hline Tl-201 Thallous Chloride & $1.40 \mathrm{E}-01$ \\
\hline Xe-133 Xenon Gas (rebreathing for 5 minutes) & 7.30E-04 \\
\hline
\end{tabular}

While it is known that thyroid uptake of radioiodine varies considerably from patient to patient [14], that the rate of hypothyroidism resulting from the treatment of Graves disease with 
radioiodine is correlated with the absorbed dose [15], and that first treatment efficacy is significantly enhanced with pretherapy planning to achieve a prescribed radiation dose [16], the debate continues over dose-based versus fixed-activity-based approaches to treatment of hyperthyroidism [17]. Treatment of thyroid cancer with ${ }^{131}$ I NaI has been a common therapeutic use of radiopharmaceuticals for many decades. Treatment regimens are generally based on a fixed-activity approach, although there have been exceptions [18, 19]. ${ }^{131}$ I metaiodobenzylguanidine (mIBG) has been used for more than 20 years for the treatment of adult and paediatric neuroendocrine tumours, including phaeochromocytoma, paraganglioma and neuroblastoma. Standard protocols involve administrations of around $7 \mathrm{GBq}$ to values over 30 GBq (for adults) [20, 21, 22]. In studies in whic dosimetry has been evaluated, it has been seen that absorbed doses to tumor, marrow and other organs vary considerably when fixed administered activities are administered [23, 24].

Two products, 'Bexxar' ( ${ }^{131}$ I-labeled tositumomab) and 'Zevalin' $\left({ }^{90}\right.$ Y-labeled Ibritumomab tiuxetan) are approved by the US FDA for treatment of relapsed or refractory Bcell non-Hodgkins lymphoma. Both agents employ the anti-CD20 antibody. Bexxar is administered after a tracer amount of the agent is given and a simple dosimetric analysis is performed that targets a whole-body dose of 0.75 Gy [25], whereas for Zevalin, dosimetry was not recommended during the approval process [26], and this agent is administered on an activity per unit body weight basis.

Skeletal Targeted Therapy (STR), mostly for palliation of pain involving bone metastases, is carried out with a number of agents, including ${ }^{89} \mathrm{Sr}$-chloride, ${ }^{153} \mathrm{Sm}$-EDTMP, (ethylenediamine tetramethylene phosphoric acid), ${ }^{186} \mathrm{Re}$ and ${ }^{188} \mathrm{Re}-\mathrm{HEDP}$ (hydroxyethylene diphosphonate), ${ }^{177} \mathrm{Lu}-177-\mathrm{MDP}$ (methylene diphosphonate) and EDTMP. The number of metastases is usually high, and dosimetry is generally not performed for use of these agents. As noted above, the introduction of alpha emitters in radiopharmaceutical therapy has caused a reevaluation of the use of appropriate radiation weighting factors to be used in internal emitter therapy. ${ }^{223} \mathrm{Ra}$ chloride has a natural affinity for metabolically active bone. It decays by the emission of $4 \alpha$-particles via progeny isotopes to stable ${ }^{207} \mathrm{~Pb}$, with a total decay energy is 28 $\mathrm{MeV}$. Hindorf et al. [27] evaluated the biodistribution and dosimetry ${ }^{223}$ Ra-chloride (Alpharadin) in patients with osteoblastic skeletal metastases secondary to hormone refractory prostate cancer. 
They found that the self absorbed dose to the kidneys was less than 0.2 Gy per injection for all patients and that the calculated absorbed doses to normal tissues following intravenous injections of ${ }^{223} \mathrm{Ra}$ chloride injections of $100 \mathrm{kBq} / \mathrm{kg}$ were very low and are unlikely to be a limiting factor in this patient population. A comprehensive evaluation of the dosimetry of ${ }^{223} \mathrm{Ra}$ chloride was recently published by Lassmann and Nosske (4). Other alpha emitters that have been or are being evaluated for radiopharmaceutical therapy (not necessarily for STR) include ${ }^{211} \mathrm{At},{ }^{212} \mathrm{Bi}$, ${ }^{213} \mathrm{Bi}$, and ${ }^{225} \mathrm{Ac}$.

The use of radiolabeled peptides for treatment of neuroendocrine tumours has been under active evaluation, using somatostatin analogues such as DOTA-DPhe(1)-Tyr(3)-octreotide (DOTATOC). Barone et al. [28] estimated kidney doses from administrations of $8-23 \mathrm{GBq}$ of ${ }^{90} \mathrm{Y}$ DOTATOC and found a strong correlation between radiation dose (expressed as 'biologically effective dose', a quantity that takes into account the rate at which dose is delivered and tissue radiosensitivity parameters and repair kinetics) and measured values of creatinine clearance in individual patients. Hindorf et al. [29] found that interpatient variability to whole body, kidneys, and tumor was quite significant, but larger than the intrapatient variability, suggesting that data obtained during a subject's first therapy may be used to guide future therapies. Forrer et al. [30] compared the biodistribution and dosimetry of ${ }^{111}$ In-DOTATOC and ${ }^{111}$ In-DOTATATE in five patients with known metastatic neuroendocrine tumors, with multiple administrations of about $200 \mathrm{MBq}$. Their analyses showed small differences between the radiopeptides, but a significantly higher mean absorbed dose to the liver was found for ${ }^{111} \mathrm{In}$ DOTATATE, and a favorable tumour-to-kidney ratio $(\mathrm{P}=0.065)$ was calculated for ${ }^{111} \mathrm{In}$ DOTATOC. A high interpatient absorbed tumor dose variability was found, which is not unexpected as receptor densities vary markedly among patients and tumors. Bodei et al. [31] evaluated long term renal toxicity after peptide radiotherapy with ${ }^{90} \mathrm{Y}$-DOTATOC and ${ }^{177} \mathrm{Lu}$ DOTATATE in 23 and 5 patients, respectively. Their results showed the high importance of the importance of clinical screening and individualized dosimetry. For patients who enter therapy with renal toxicity-associated risk factors, patients should not receive a BED higher than 28 Gy, and noted that fractionation of activity in multiple cycles is also helpful in managing renal toxicity. Barone et al. [32] evaluated kidney toxicity in the administration of ${ }^{90} \mathrm{Y}$-DOTATOC in nine male and nine female subjects. Estimation of renal dose to observe and control possible 
toxicity were significantly influenced by distribution of the radionuclide, size of the target organ (i.e., the kidney), the absorbed dose rate and fractionation.

\section{CONCLUSIONS}

The science of internal dosimetry in nuclear medicine is in a very mature state. Many resources for facilitating dose calculations are available, and internet and software tools have allowed many users to perform dosimetry calculations, once appropriate biokinetic data are gathered in animal or human experiments. A considerable number of diagnostic and therapeutic radiopharmaceuticals are widely available; the number and type of agents is frequently changing, so new dosimetry calculations are always being needed. A most interesting recent development is the appearance of alpha emitters for use in radiopharmaceutical therapy, and many promising new therapeutic agents are being tested and introduced to the worldwide market. 


\section{REFERENCES}

1. Marinelli L, Quimby E, Hine G: Dosage determination with radioactive isotopes II, practical considerations in therapy and protection, Am J Roent Radium Ther 59:260-280, 1948.

2. Quimby E and Feitelberg S: Radioactive isotopes in medicine and biology, Philadelphia, 1963, Lea and Febiger.

3. Bodey RK, Flux GD, Evans PM. Combining dosimetry for targeted radionuclide and external beam therapies using the biologically effective dose. Cancer Biother Radiopharm. Feb;18(1):89-97, 2003.

4. Lassmann M, Nosske D. Dosimetry of 223Ra-chloride: dose to normal organs and tissues. Eur J Nucl Med Mol Imaging. Jan;40 (2):207-12, 2013.

5. Stabin MG,Siegel JA. Physical Models and Dose Factors for Use in Internal Dose Assessment. Health Physics, 85 (3):294-310, 2003.

6. Stabin MG, Sparks RB, Crowe E. OLINDA/EXM: The Second-Generation Personal Computer Software for Internal Dose Assessment in Nuclear Medicine. J Nucl Med 46 1023-1027, 2005.

7. Cristy M. and Eckerman K. Specific absorbed fractions of energy at various ages from internal photons sources. ORNL/TM-8381 V1-V7. Oak Ridge National Laboratory, Oak Ridge, TN, 1987.

8. Stabin M, Watson E, Cristy M, Ryman J, Eckerman K, Davis J, Marshall D., Gehlen K. Mathematical models and specific absorbed fractions of photon energy in the nonpregnant adult female and at the end of each trimester of pregnancy. ORNL Report ORNL/TM-12907, 1995.

9. Stabin MG, Xu XG, Emmons MA, Segars WP, Shi C, Fernald MJ. RADAR Reference Adult, Pediatric, and Pregnant Female Phantom Series for Internal and External Dosimetry, J Nucl Med 253:1807-1813, 2013.

10. Stabin MG. Fundamentals of Nuclear Medicine Dosimetry. Springer, New York, NY, February 2008. 
11. Siegel J, Thomas S, Stubbs J, Stabin M, Hays M, Koral K, Robertson J, Howell R, Wessels B, Fisher D, Weber D, Brill A. MIRD Pamphlet No 16 - Techniques for Quantitative Radiopharmaceutical Biodistribution Data Acquisition and Analysis for Use in Human Radiation Dose Estimates. J Nucl Med 40:37S-61S, 1999.

12. International Commission on Radiological Protection. Radiation Dose Estimates for Radiopharmaceuticals. ICRP Publications 53, 80, and 106, with addenda. Pergammon Press, 1983-2008.

13. International Commission on Radiological Protection. Recommendations of the ICRP. ICRP Publication 103, Annals of the ICRP 37 (2-3) 2007.

14. Eschmann, S. M., Reischl, G., Bilger, K., Kupferschlager, J., Thelen, M. H., Dohmen, B. M., Besenfelder, H., \& Bares, R. "Evaluation of dosimetry of radioiodine therapy in benign and malignant thyroid disorders by means of iodine-124 and PET", European Journal of Nuclear Medicine and Molecular Imaging, vol. 29, no. 6, pp. 760-767, 2002.

15. Grosso M, Traino A, Boni G, Banti E, Della Porta M, Manca G, Volterrani D, Chiacchio S, AlSharif A, Borso E, Raschilla R, Di Martino F, Mariani G. Comparison of different thyroid committed doses in radioiodine therapy for Graves' hyperthyroidism. Cancer Biotherapy And Radiopharmaceuticals. 20(2) 218-223, 2005.

16. Kobe C, Eschner W, Sudbrock F, Weber I, Marx K, Dietlein M, Schicha H. Graves' disease and radioiodine therapy: Is success of ablation dependent on the achieved dose above 200 Gy? Nuklearmedizin, 47 1: 13-17, 2008.

17. Stabin MG, Flux GD. Internal dosimetry as a tool for radiation protection of the patient in nuclear medicine. Review article, Biomedical Imaging and Intervention Journal, 3(2):e28, 2007.

18. Furhang E. E., Larson S. M., Buranapong P., \& Humm J. L. "Thyroid cancer dosimetry using clearance fitting", Journal of Nuclear Medicine, vol. 40, no. 1, pp. 131-136, 1999.

19. Maxon H. R., Thomas S. R., \& Samaratunga R. C. "Dosimetric considerations in the radioiodine treatment of macrometastases and micrometastases from differentiated thyroid cancer", Thyroid, vol. 7, no. 2, pp. 183-187, 1997.

20. Hoefnagel C. A. "Nuclear medicine therapy of neuroblastoma", Quarterly Journal of Nuclear Medicine, vol. 43, no. 4, pp. 336-343, 1999. 
21. Matthay K. K., Panina C., Huberty J., Price D., Glidden D. V., Tang H. R., Hawkins R. A., Veatch J., \& Hasegawa B. "Correlation of tumor and whole-body dosimetry with tumor response and toxicity in refractory neuroblastoma. treated with I-131-MIBG", Journal of Nuclear Medicine, vol. 42, no. 11, pp. 1713-1721, 2001.

22. Gaze M. N., Chang Y. C., Flux G. D., Mairs R. J., Saran F. H., \& Meller S. T. "Feasibility of dosimetry-based high-dose I-131-meta-iodobenzylguanidine with topotecan as a radiosensitizer in children with metastatic neuroblastoma", Cancer Biotherapy and Radiopharmaceuticals, vol. 20, no. 2, pp. 195-199, 2005.

23. Monsieurs M., Brans B., Bacher K., Dierckx R., \& Thierens H. "Patient dosimetry for I-131MIBG therapy for neuroendocrine tumours based on I-123-MIBG scans", European Journal of Nuclear Medicine and Molecular Imaging, vol. 29, no. 12, pp. 1581-1587, 2002.

24. Flux G. D., Guy M. J., Papavasileiou P., South C., Chittenden S. J., Flower M. A., \& Meller S. T. "Absorbed dose ratios for repeated therapy of neuroblastoma with I-131 mIBG", Cancer Biotherapy and Radiopharmaceuticals, vol. 18, no. 1, pp. 81-87, 2003.

25. Wahl R. L., Kroll S., \& Zasadny K. R. "Patient-specific whole-body dosimetry: Principles and a simplified method for clinical implementation", Journal of Nuclear Medicine, vol. 39, no. 8, pp. 14S-20S, 1998.

26. Wiseman G. A., White C. A., Sparks R. B., Erwin W. D., Podoloff D. A., Lamonica D., Bartlett N. L., Parker J. A., Dunn W. L., Spies S. M., Belanger R., Witzig T. E., \& Leigh B. R. "Biodistribution and dosimetry results from a phase III prospectively randomized controlled trial of Zevalin (TM) radioimmunotherapy for low-grade, follicular, or transformed B- cell non-Hodgkin's lymphoma", Critical Reviews in Oncology Hematology, vol. 39, no. 1-2, pp. 181-194, 2001.

27. Hindorf C, Flux G, Lewington V, Aksnes AK, Harris P, Parker C. A biodistribution and dosimetry study of therapeutic 223Ra-chloride (Alpharadin) in patients with osteoblastic skeletal metastases secondary to hormone refractory prostate cancer. J Nucl Med. 49 (Supplement 1):145P, 2008.

28. Barone R., Walrand S., Valkema R., Kvols L., Smith C., Krenning E. P., Jamar F., \& Pauwels S. "Correlation between acute red marrow (RM) toxicity and RM exposure during Y-90-SMT487 therapy", Journal of Nuclear Medicine, vol. 43, no. 5, p. 1267, 2002. 
29. Hindorf, C., Chittenden, S., Causer, L., Lewington, V. J., Macke, H. R., Flux, G. D Dosimetry for Y-90-DOTATOC therapies in patients with neuroendocrine tumors. Cancer Biother Radiopharm, 22 (1):130-135, 2007.

30. Forrer F, Uusijärvi H, Waldherr C, Cremonesi M, Bernhardt P, Mueller-Brand J, Maecke. HR. A comparison of 111In-DOTATOC and 111In-DOTATATE: biodistribution and dosimetry in the same patients with metastatic neuroendocrine tumours. Eur J Nucl Med Mol Imaging 31:1257-1262, 2004.

31. Bodei L, Cremonesi M, Ferrari M, Pacifici M, Grana CM, Bartolomei M, Baio SM, Sansovini M,. Paganelli G. Long-term evaluation of renal toxicity after peptide receptor radionuclide therapy with 90Y-DOTATOC and 177Lu-DOTATATE: the role of associated risk factors. Eur J Nucl Med Mol Imaging 35:1847-1856, 2008.

32. Barone R, Borson-Chazot F, Valkema R, Walrand S, Chauvin F, Gogou L, Kvols LK, Krenning EP, Jamar F, Pauwels S. Patient-Specific Dosimetry in Predicting Renal Toxicity with 90Y-DOTATOC: Relevance of Kidney Volume and Dose Rate in Finding a DoseEffect Relationship. J Nucl Med 46:99S-106S, 2005. 